\title{
A Sharpening Algorithm for Dynamic Video Image in Network Education
}

\author{
Huixian LI, Linhan LI
}

Hebei Finance University, Hebei, China

\begin{abstract}
In distance education by network, the dynamic images include a lot of noise and bad data. This paper presents an image sharpness algorithm based on pixel small group enhancement. Firstly it adopts discrete wavelet transform method to filtrate the noise. And secondly the pixel small group principle is used to enhance the image and guarantee the clearance and liability of video images. Experimental results show that the new method improves the image quality and it is more effective than the traditional method.
\end{abstract}

KEYWORD: video education; pixel group; image sharpness

\section{INTRODUCTION}

Modern distance education has become an important part of higher education in china as a new mode of teaching [1-4]. In Network Education, the image signals have to pass a long distance and suffer a tremendous attenuation. The dynamic video images have got serious noise, thus making the picture blurry [5-7]. The neural network and ant colony algorithm methods are frequently used for image sharpness. But there is a lot of nonwhite noise in the remote images. The traditional image de-noising methods cannot build up a dynamical analysis model for the multidimensional noise characteristic, so the interference factors can't be eliminated and the image quality is damaged [8] This paper presents an image sharpness algorithm based on pixel small group enhancement. The algorithm adopts discrete wavelet transform method to filtrate the noise. Then the pixel small group principle is used to enhance the image and ensure the clarity of motion blurred image. Simulation experiments show the validity of the proposed method.

\section{A SHARPENING ALGORITHM FOR DYNAMIC IMAGE}

\subsection{Wavelet transform for image de-noising}

Web-based course is an important component of educational resource. There is a lot of digital images and video information in network teaching resource [9-10]. And the images can form an image sequence which is denoted by $\mathrm{s}(\mathrm{u})$. A transform pair of the image sequence can be obtained with the formula below.

$$
\begin{aligned}
& U_{\xi}\left(j_{1}, k\right)=\frac{1}{\sqrt{M}} \sum_{v} s(u) \mu_{j_{1}, k}(u) \\
& U_{\xi}(j, k)=\frac{1}{M} \sum_{v} s(s) \xi_{j, k}(u)
\end{aligned}
$$

If it satisfies $\mathrm{j} \geq \mathrm{j}_{0}$, so:

$$
s(u)=\frac{1}{M} \sum_{k} U_{\mu}\left(j_{0}, k\right) \mu_{j_{0}, k}(u)+\frac{1}{\sqrt{M}} \sum_{j=j_{0}} \sum_{k} U_{\mu}(j, k) \xi_{j, k}(u)
$$

In the formula above, $\mathrm{s}(\mathrm{u}), \mu_{\mathrm{j} 0, \mathrm{k}}(\mathrm{u})$ and $\zeta_{\mathrm{j}, \mathrm{k}}(\mathrm{u})$ are all the function of discrete variables $u=1,2, \cdots, M$.A stochastic sample of $\mathrm{u} 0, \Delta \mathrm{u}$ is selected. $s(u)=s\left(u_{0}, u \Delta u\right)$. Let $\mathrm{j}_{0}=0$ and $\mathrm{M}=2$, then sum up $u=1,2, \cdots, M, j=1,2, \cdots, J$ and $k=1,2, \cdots, 2^{n}-1$. Four pixels are selected randomly: $s(0)=1$, $s(1)=4, s(2)=-3$ and $s(3)=0$. Then sum up as $u=1,2,3,4, \quad j=1,2$. It can be obtained that

$U_{\mu}(0,0)=\frac{1}{2} \sum_{u=1}^{4} s(u) \mu_{0,0}(u)=\frac{1}{2}[1 \cdot 1+4 \cdot[-3 \cdot 1+1 \cdot 1]=2$

The above-mentioned method can be used to ensure the equilibrium of sample images. The sample images with similar interval can be collected with the formula below.

$U_{\xi}(0,0)=\frac{1}{2}[2 \cdot 2+4 \cdot(-3)+1 \cdot 0+(-1) \cdot 4]=-6$ 
$U_{\xi}(1,1)=\frac{1}{2}[2 \cdot \sqrt{3}+1 \cdot(-\sqrt{3})+2 \cdot 1+1 \cdot 1]=\frac{3+\sqrt{3}}{2}$

$U_{\xi}(1,0)=\frac{1}{2}[2 \cdot 2+0 \cdot 2+1 \cdot 1+1 \cdot 2]=\frac{7}{2}$

Then the image is decomposed by discrete wavelet transform with the formula below.

$$
\begin{aligned}
s(u)= & \frac{1}{2}\left[U_{\mu}(0,0) \mu_{0,0}(v)+U_{\mu}(0,0) \mu_{0,0}(u)\right. \\
& \left.+U_{\xi}(1,0) \xi_{1,0}(u)+U_{\xi}(1,1) \xi_{1,1}(u)\right]
\end{aligned}
$$

So the interference factors are filtered out and the image quality is improved.

\subsection{Pixel group feature enhancement}

Based on the above analysis, it can be seen that the relationship between the image characteristics is the prerequisite of image enhancement. However, the pixel feature relationship in education image shows an obvious diversity and random. So the energy function is adopted to construct the relationship between frames. The energy function mainly is used for analyzing and calculating the likelihood energy and the prior energy of image feature. The likelihood energy is used to describe the interference factors in the characteristics of the former frame. The prior energy is used to predict the compression of frames in different image. When constructing the prior energy, the local constraints are to be melted. By analyzing the image features of video education, the likelihood energy and the prior energy Markova random field are constructed to obtain the objective function.

\subsubsection{The posterior energy}

In the neighborhood system of the current image and the prior image of the frame sequence, the posterior energy $W(\beta / E)$ includes the likelihood energy $\mathrm{W}(\mathrm{E} / \beta)$ and the prior energy $\mathrm{W}(\beta)$.

$W(\beta / E)=d_{1} \times W(E / \beta)+W(\beta)$

In the formula above, $\mathrm{d} 1$ represents the weight coefficient. The target function of the proposed algorithm is the posterior energy $W(E / \beta)$.By estimating the minimum value of the posterior energy, the predictive maximum value can be obtained.

$$
\beta^{*}=\arg \min _{R} W(\beta / E)
$$

In the formula above, $\beta *$ represents the position of pixel feature points to be enhanced.

\subsubsection{The likelihood energy}

In moment $\mathrm{t}$, the projection in the imaging plane of feature point $\mathrm{i}$ in different frames can be described by the formula below.

$$
E_{i}^{t}=E_{i}^{t^{\prime}}+z_{i}^{t}
$$

Among the formula $E_{i}^{t}=\left(u_{i}^{t}, v_{i}^{t}\right)$ can be used to describe the practically observed projection point of network features. $E_{i}^{t^{\prime}}=\left(u_{i}^{t^{\prime}}, v_{i}^{t^{\prime}}\right)$ can be used to describe the projection point of features by computation.$z_{i}^{t}$ describes the interference of random noise. If the noise term $z_{i}^{t}=\left(z_{i u}^{t}, v_{i v}^{t}\right)$ fits the Gaussian distribution, we can conclude that $z_{u i}^{t} \sim Z\left(0, \eta^{2}\right)$ and $z_{v i}^{t} \sim Z\left(0, \eta^{2}\right)$.In this paper, we assume that there is not interference in noise distribution of feature points in different frames of video, and the noise distribution does not link up with each other on time. The conditional probability of the plane coordinate of imaging point can be described by the formula below.

$$
\begin{aligned}
& E(E / \beta)=\prod_{t=1}^{n} \prod_{i=1}^{m} E\left(E_{i}^{t} / \beta_{i}, t\right) \\
& E\left(E_{i}^{t} / \beta_{i}, t\right)=\frac{1}{\sqrt{2 \pi}} \exp \left[-\frac{\left(u_{i}^{t}, u_{i}^{t^{\prime}}\right)^{2}+\left(v_{i}^{t}, v_{i}^{t^{\prime}}\right)^{2}}{2 \eta^{2}}\right]
\end{aligned}
$$

The parameter $E=\left\{E_{i}^{t} \mid i \in B, t=1,2, \cdots, M\right\}$ can be used to describe the set of coordinate of images plane of point of features represents the number of collected frames. So we can obtain the likelihood energy of image feature expressed by the formula below.

$$
E(E / \beta)=\frac{1}{2 \eta^{2}} \sum_{t=1}^{G} \sum_{i=1}^{M}\left[\left(u_{i}^{t}-u_{i}^{t^{\prime}}\right)^{2}+\left(v_{i}^{t}-v_{i}^{t^{\prime}}\right)^{2}\right.
$$

The potential energy of position i in pixel small group can be obtained by penalty function, as shown below.

$$
W_{2}\left(\mu_{i}, \beta_{i}\right)=\min \left\{\left\|\beta_{i}-\beta_{i^{\prime}}\right\|^{2}, \varepsilon\right\}
$$

In the formula, $\varepsilon$ is a threshold above zero, it reflects the correlation degree between the fuzzy varying parameter of adjacent characteristic points which can be used to enhance the detail definition in the image. 


\section{SIMULATION RESULTS}

By analyzing simulation result, every methods impact on network image quality is shown. The neural network and ant colony algorithm methods are taken for image processing compared with the proposed algorithm. The original image is shown below.

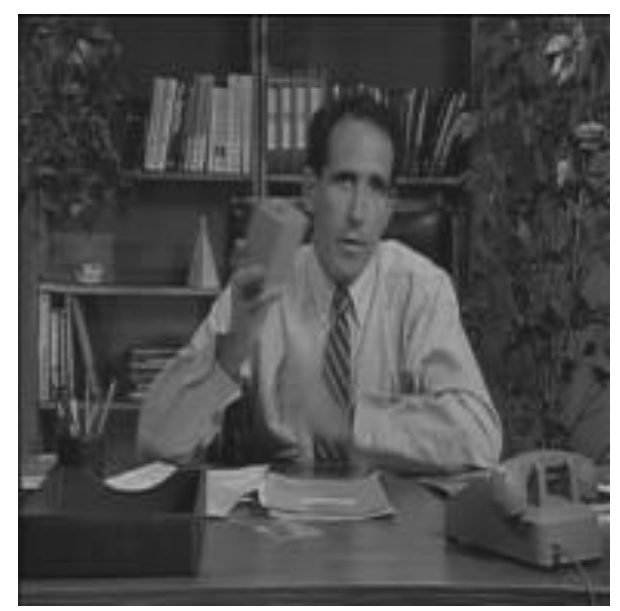

Figure 1. The original image

The image with noise is shown below.

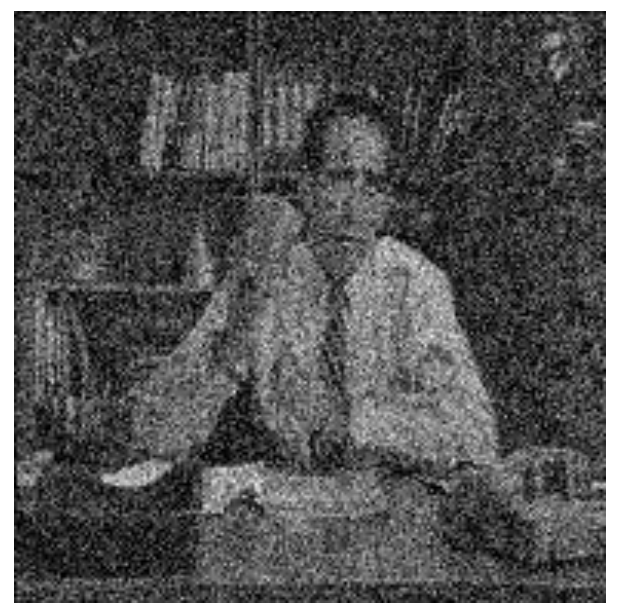

Figure 2. The image with noise

The result by the neural network is shown below.

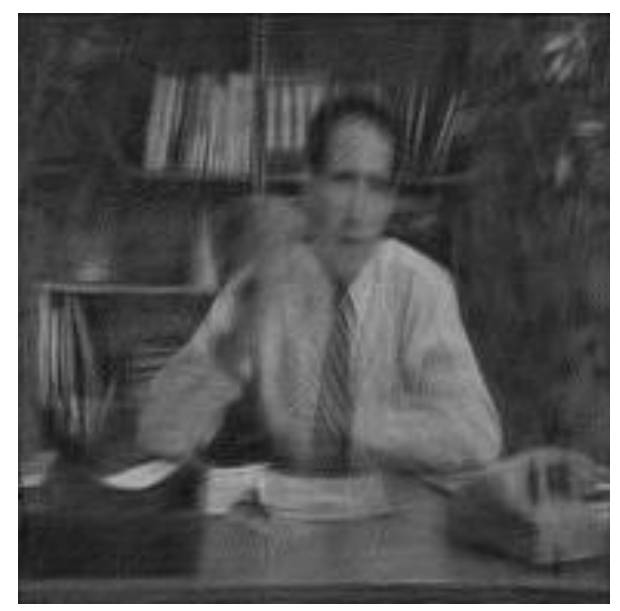

Figure 3. The result by the neural network
The result by the ant colony algorithm method is shown below.

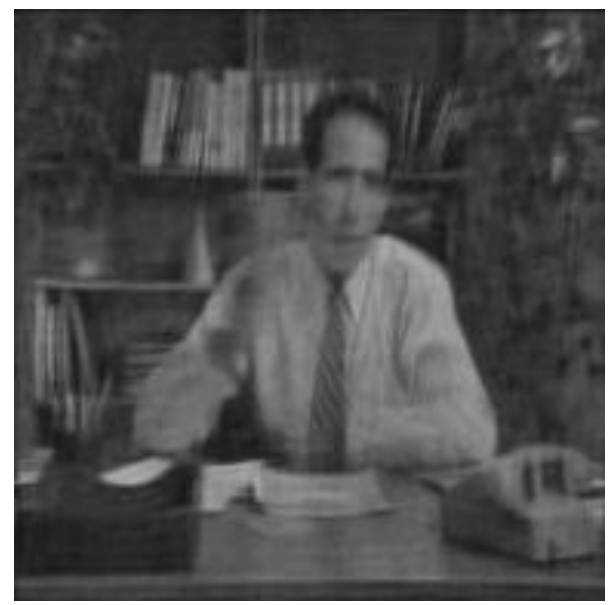

Figure 4 . The result by the ant colony algorithm

The result by the proposed algorithm is shown below.

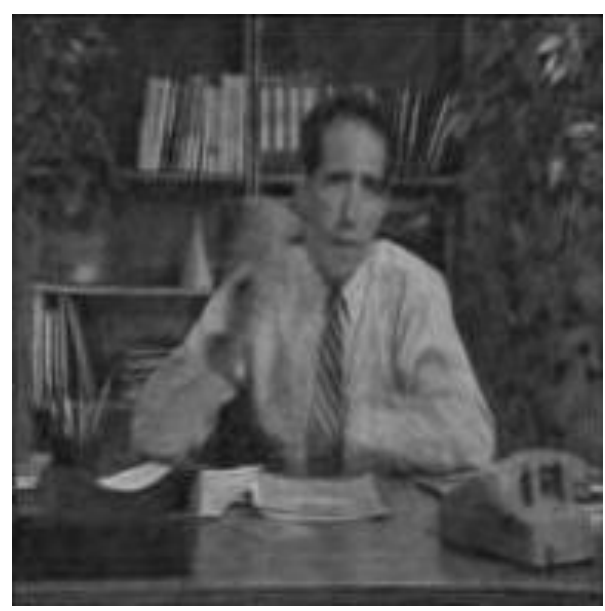

Figure 5 . The result by the proposed algorithm

It can be seen that there are scratch and smear in the result by the neural network. There is borderline blurred and double shadow phenomenon in the result by the ant colony algorithm. The image processing result of the proposed algorithm also has wailing smear, but the interference is slight. This method preserves the edge information and inhibits the noise effectively. The figure below shows the comparison of PSNR. 


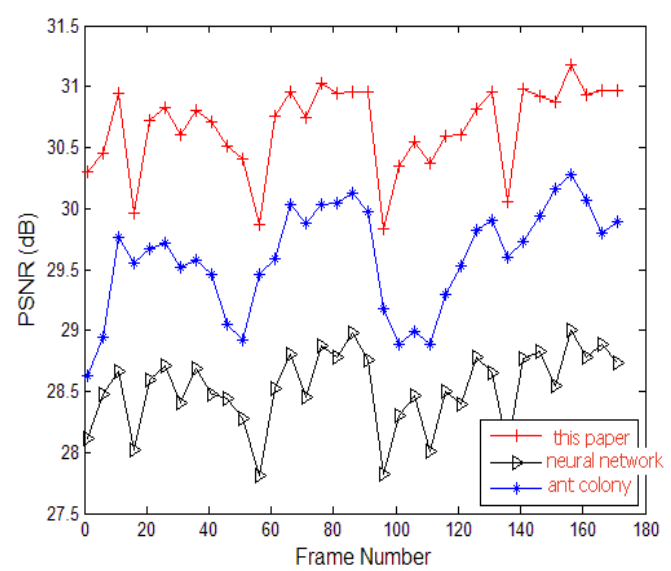

Figure 6. The comparison of PSNR

From the figure, it can be seen that this new algorithm yields better results, both visually and in terms of PSNR are better than the other two methods. Experimental results demonstrate that the proposed algorithm is more advantageous than traditional algorithms in accuracy and efficiency.

\section{CONCLUSION}

In This paper, we present an image sharpness method based on pixel small group enhancement. It adopts discrete wavelet transform method and the pixel small group principle to enhance the image. Experimental results show that the proposed method is effective. For distance learning, its survival and continuity development depends on its image quality. The proposed algorithm is simple, stable and highly portable, and has bright future of wide application.

\section{REFERENCES}

[1] Y. M. Lu and M. N. Do. Multidimensional nonsubsampled hourglass filter banks: geometry of passband support and filter design. Fortieth Asilomar Conference on Signals, Systems and Computers. 2014, 406-410.

[2] E. Chang and A. Zakhor. Subband video coding based on velocity filers. IEEE International Symposium on Circuits and Systems. 2012, 2288-2291.

[3] Y. B. Chen, X. Y. Guo and W. M. Shen. Robust adapgtive spatio-temporal video denoising algorithm based on motion estimation. Computer Applications. 2014, 26(8):1882-1 887.

[4] F. A. Mujica, J. P. Leduc, R. Murenzi, et al. A new motion parameter estimation algorithm Based on the continuous wavelet transform. IEEE Transactions on Image Processing. 2000, 9(4):873-888.

[5] M. N. Do and M. Vetterli. The contourlet transform: An efficient directional multiresolution image representation. IEEE Transactions on Image Processing. 2014, 14(12): 2091-2105.

[6] Y. M. Lu and M. N. Do. Multi-dimensional directional filter banks and surfacelets. IEEE Transactions on Image Processing. 2007, 16(4):918-931.

[7] Y. M. Lu and M. N. Do. 3-D directional filter banks and surfacelets. IEEE Transactions on Image Processing. 2007, 16(4):918-931.

[8] Y. M. Lu and M. N. Do. Video processing using the 3Dimensional Surfacelet transform. Fortieth Annual Asilomar Conference on Signals, Systems and Computers. 2014, 883-887.

[9] Y. M. Lu. Multidimensional geometrical signal representation: constructions and applications. Ph.D. dissertation, University of Illinois at Urbana-Champaign, Urbana.2007.

[10] Y. M. Lu and M. N. Do. A mapping based design for nonsubsampled hourglass filter banks in arbitrary dimensions. IEEE Transactions on Signal Processing. 2014, 56(4): 1466-1478. 